\title{
Philosophiques
}

\section{Le pseudo-féminisme du néo-conservatisme féminin}

\section{Michel Lavoie}

Volume 21, numéro 2, automne 1994

Les femmes et la société nouvelle

URI : https://id.erudit.org/iderudit/027278ar

DOI : https://doi.org/10.7202/027278ar

Aller au sommaire du numéro

Éditeur(s)

Société de philosophie du Québec

ISSN

0316-2923 (imprimé)

1492-1391 (numérique)

Découvrir la revue

Citer ce document

Lavoie, M. (1994). Le pseudo-féminisme du néo-conservatisme féminin. Philosophiques, 21(2), 357-364. https://doi.org/10.7202/027278ar

\section{Résumé de l'article}

Le féminisme a réussi à transformer partiellement le monde dans lequel nous vivons. Mais la résistance de la société traditionnelle à ces changements, ainsi que la remontée de la droite et la valorisation forcenée de l'individualisme, risquent de compromettre ses acquis. Dans ce contexte, l'avènement d'un discours pseudo-féministe qui véhicule les valeurs traditionnellement considérées comme " féminines " par les hommes risque d'embrouiller le message féministe pour tous ceux qui y accordaient créance et espéraient le voir aller plus loin dans sa quête d'une société nouvelle. Nous voulons analyser ce discours en montrant comment il tend à remettre en question l'effort de transformation sociale amorcé par le féminisme. 


\title{
Le pseudo-féminisme du néo-conservatisme féminin
}

\author{
par \\ Michel Lavoie
}

\begin{abstract}
RESUME : Le féminisme a réussi à transformer partiellement le monde dans lequel nous vivons. Mais la résistance de la société traditionnelle à ces changements, ainsi que la remontée de la droite et la valorisation forcenée de l'individualisme, risquent de compromettre ses acquis. Dans ce contexte, l'avenement d'un discours pseudo-féministe qui véhicule les valeurs traditionnellement considérées comme "féminines » par les hommes risque d'embrouiller le message féministe pour tous ceux qui y accordaient créance et espéraient le voir aller plus loin dans sa quête d'une société nouvelle. Nous voulons analyser ce discours en montrant comment il tend à remettre en question l'effort de transformation sociale amorcé par le féminisme.
\end{abstract}

\begin{abstract}
Feminism has partly transformed our world. Traditionnal society's resistance to these changes together with the rise of the Right and individualism endanger these gains. In that context, the advent of pseudo-feminist discourse carrying the values traditionnally seen by men as « féminine » might blur the feminist message for those who give it credit and hope to see it progress in its quest for a new society. We wish to analyse that discourse and show how it tends to challenge the social transformation effort launched through feminism.
\end{abstract}

Il n'est pas facile en tant qu'homme de se prononcer sur la situation de la femme. Mais d'un point de vue hétéropolitique, le féminisme représente une idéologie de libération, et il n'est pas besoin, pour y adhérer, de faire référence à une différence hormonale. En ce sens le féminisme est aussi le lieu des hommes qui croient que «l'égalité » n'est pas un mot vide de sens.

La présentation qui va suivre se veut résolument pamphlétaire; elle se veut aussi une dénonciation d'une situation dangereuse et qui menace l'équilibre fragile qui tente de s'établir. Cet équilibre pourrait éventuellement nous mener à l'établissement d'une société réellement égalitaire et juste établie sur des bases nouvelles et qui ne retiendrait des rôles hommes/femmes traditionnels que l'injustice historique qu'ils représentent. En utilisant le même discours qu'une soi-disant élite, un langage qui peut éventuellement sembler pour le moins dérangeant, nous tenterons de mettre en évidence ce 
qui nous paraît être une intense tentative de récupération de la pensée féministe, qui perdrait ainsi, vidée de son sens profond d'idéologie de libération, tout caractère révolutionnaire et original. C'est ainsi que je veux vous entretenir de ce que l'on peut appeler «le pseudo-féminisme du néoconservatisme féminin ».

La remontée inquiétante des idéologies de droite, notamment chez nos voisins du Sud (U.S.A.), conditionne de plus en plus le discours social. Le nouveau credo est essentiellement d'ordre économique, au détriment du choix de société. La « rentabilité » devient le commun dénominateur, et la sagesse de «l'homme d'affaires » nous fait prendre conscience de l'inutilité des changements qui pourraient créer un climat d'insécurité néfaste pour le marché. Cela pourrait éventuellement nous coûter nos précieux emplois et donner une image d'instabilité aux investisseurs (en majorité états-uniens) qui craignent continuellement que le Québec bascule dans le communisme. En effet, de nombreux indices « inquiétants » montrent que notre société peut facilement devenir une enclave socialiste dans le bastion de la liberté (lire libre entreprise et/ou «American way of life ») qu'est le continent nord-américain. L'assurance-maladie, l'assurance-auto, les offices de mise en marché agricoles, les Caisses Populaires, la Caisse de dépôts et placements du Québec, et j'en passe, tous ces organismes, par leur caractère « universalisant », contribuent à véhiculer au Québec des idées aussi « monstrueuses » que l'égalitarisme et la recherche d'une plus grande qualité de vie, sinon d'une vie de qualité.

Le féminisme n'échappe pas à cette épuration nécessaire que notre collectivité devra effectuer. Afin de pouvoir conserver un accès au marché états-unien, nous avons à faire la preuve de notre profonde adhésion aux « valeurs » traditionnelles qui ont permis à notre grand frère du sud de s'élever au rang de leader mondial, et ce avec la « Bénédiction Divine » qui devrait constituer une preuve incontestable de son «bon droit ». Effectivement, le féminisme est dangereux pour l'ordre et l'équilibre nécessaires aux affaires.

Les revendications du genre «à travail égal, salaire égal », ou de conditions de travail appropriées, de congés décents de maternité-paternité, d'un système éducationnel adéquat/cohérent/gratuit, d'un tissu social juste et valorisant l'épanouissement des citoyennes/citoyens, sont directement issues du discours féministe et du militantisme. Toutes ces choses «jugées bonnes en soi » sont également considérées utopiques, dans le sens péjoratif du terme. En bousculant l'ordre traditionnel TravaIL-FAMILIE-PATRIE et la hiérarchie paternelle/paternaliste, ces propositions de restructuration sociale imposent des contraintes au milieu des affaires. Un meilleur partage des richesses collectives implique une « perte de profits » qui pourrait être néfaste à la santé de nos entreprises (et peut-être aussi à celle de nos entrepreneurs), c'est du moins ce que le corрогаtisme capitaliste voudrait nous faire croire.

La mauvaise influence de la pensée féministe se fait de plus en plus sentir : on voit le «masculin » perdant du terrain et le paternalisme considéré comme une mentalité réactionnaire et aliénante. Le discours officiel lui-même se voit 
imposer une adhésion « contre nature » à ces nouvelles valeurs qui nient ce qui a fait que notre société est ce qu'elle est. Les hommes (mâles) commencent à s'habituer à cette situation et la perçoivent comme une nette amélioration. Le harcèlement sexuel est aujourd'hui considéré inacceptable par une grande majorité, le partage des tâches s'étend, l'égalitarisme fait du chemin; une tendance s'est amorcée qui se voulait irréversible. Les effets du militantisme féministe des années 1960-1970-1980 se font enfin sentir de façon notable.

Personnellement, en tant « qu'homme » (mâle), je ne voudrais jamais voir revenir la situation traditionnelle de la femme. Mais, dans un certain milieu conservateur, de nombreux vrais hommes ne partagent pas cet avis. Ce conservatisme se manifeste ouvertement, maintenant que la politique se fait entre tenants des « valeurs traditionnelles » et « ultra-conservateurs », supportés par les pouvoirs financiers qui trouvent naturellement leur intérêt dans un retour à la stabilité.

Dois-je considérer que je ne suis pas un vrai homme? Mon inconfort personnel face à cette résurgence de la droite est peut-être le signe de mon incapacité à vivre dans une société «dynamique » où le vendeur agressif constitue un élément positif, face aux inutiles que sont les chômeurs, les assistés sociaux et tous les pauvres qui, par leur condition même, manifestent ainsi leur « mentalité de perdants ». Je considérais que l'évolution était un courant irrésistible et que la situation, pour un peu qu'on l'aide, ne pouvait que continuer à tendre vers ce que je croyais être un mieux-être. La montée du féminisme, comme idéologie et mouvement de libération, me semblait être cette évolution que l'humanité attendait. Or il semble qu'avec beaucoup d'autres, je me trompais.

C'est un certain discours de femmes qui m'a fait prendre conscience de mon erreur. Condamné, en tant que philosophe, à l'écriture et à l'écoute, j'essaie de me tenir à jour. Assis à ma table de travail, cherchant dans mon ordinateur une présence amicale stimulant une certaine émulation, je garde contact avec l'extérieur par le biais de la radio. J'ai ainsi « l'avantage » de passer un certain nombre de journées à écouter, en travaillant, ce qui se véhicule dans notre société. Je syntonise notre officielle Radio d'État (Radio-Canada), sur le réseau A.M., et profite ainsi d'un déversement continu d'informations « éclairées » (par l'idéologie en place), de « sagesse » démystifiée (j'écoute Jacques Languirand), et de « divertissements » intellectuels d'un éclectisme retentissant : j'écoute Mmes Christiane Charette et Marie-France Bazzo.

J'ai donc le bénéfice de recevoir, dans ma fiévreuse atmosphère de travail, le nec plus ultra d'un discours qui se dit à l'avant-garde et relevant des nouveaux courants du féminisme actuel, héritage direct du militantisme des trois dernières décennies. Si cela est juste j'aimerais mieux, à l'instar de l'imposante gallinacée que l'on appelle l'autruche, enfouir ma pauvre tête dans le sable et chercher un oubli bienfaisant dans ma croyance en un féminisme révolutionnaire et engagé. Où sont-elles celles qui croyaient que le monde pouvait être changé ? 
La puérilité du discours véhiculé dans ces émissions n'a d'égal que l'incroyable retour en arrière qu'il constitue et semble même valoriser. Pour un homme (mâle), qui s'évertue à découvrir les nouvelles modalités de vie que le féminisme militant nous avait amené à conscientiser, la résurgence des rôles traditionnels comme « champ définitionnel » de la femme moderne est pour le moins stupéfiante. J'ai donc décidé de prendre parti.

Quand j'ai entendu, pour la première fois, Mme Bazzo et les gens de son équipe, faire l'apologie de ce qu'elles nomment «la néo-poupoune »,j'ai cru à une blague. Je ne pouvais sincèrement croire qu'encore à notre époque des femmes, qui plus est des femmes ayant une aussi grande audience publique, pouvaient sciemment emprunter les moyens débilitants de la « réaction » pour discuter de la condition de la femme moderne. Quand j'ai constaté qu'à plusieurs reprises elles se targuaient de représenter le courant néo-poupouniste qui, selon elles, correspondait à la réalité de la femme des années ig9o, j'en suis resté estomaqué.

En écoutant régulièrement les émissions de Mmes Bazzo et Charette, j'ai constaté qu'elles ne font que transmettre une certaine vision de la femme qui correspond à l'idéologie d'une société reprise en main par le pouvoir corporatiste de droite, à l'image de ce qui se véhicule aux U.S.A. La « néo-poupoune » est une femme qui se veut à l'aise dans la société, relativement jeune, relativement jolie, et assez bien nantie; si de plus elle a un « chum » yuppie tout est complet. Cela constitue une certaine idéalisation de la « petite bourgeoise » qui s'amuse, qui lit, qui se cultive, mais dont l'action ne porte jamais à conséquence bien longtemps. Cette femme devient une espèce de « caniche »: enjouée et espiègle, de bonne compagnie, pouvant éventuellement montrer les dents mais que personne ne prend réellement au sérieux, toujours bien «brossée et taillée », et dont la vie se déroule, sans heurt, dans une perpétuelle atmosphère bon enfant. Dotée de confortables revenus, cette femme peut satisfaire toutes ses envies, jamais elle ne prend conscience de ce qui l'entoure autrement que par ce qui fait également partie de son monde. En petit tailleur griffé, cette femme traverse la vie au volant de son amusante petite voiture, entretenue à grands frais par un « chum » bienveillant qui lui passe toutes ses petites « folies», entourée par tous les derniers gadgets à la mode et dont « personne » ne saurait vraiment se passer. Le « chum », lui, est généralement quelqu'un d'important. Il est juste assez sportif pour entretenir la « forme », juste assez préoccupé par ses affaires pour laisser une certaine liberté à sa « créature », et juste assez viril pour que ce soit amusant.

Pour la néo-poupoune, l'univers masculin est essentiellement divisé en deux parties irréconciliables : d'un côté l'homme rose, de l'autre le macho. L'homme rose se doit d'être gentil, sensible, pouvant même pleurer, à l'écoute, capable de vivre intensément ses émotions et son aspect féminin; en fait il serait parfait s'il n'était parfois morne et ennuyeux comme amant... Le macho, lui, est porteur de tous les défauts « inhérents » à la nature masculine traditionnelle, mais il est si séduisant et baise si bien... 
« L'éternel féminin » de la néo-poupoune se débat sans espoir devant cette dichotomie masculine qui lui occasionne un perpétuel déchirement: choisir entre un «bon mari » rose, dispensant sécurité et aisance financière, et un amant macho, distribuant plaisir et situations surprenantes qui brisent si bien la routine. La néo-poupoune vit dans un univers empreint de romantisme traditionnel modernisé, où le cheval blanc est remplacé par la voiture de sport ou de luxe, mais dans lequel elle se déplace en voiture familiale, conséquence d'une « saine » planification financière, quand elle choisit finalement une sécurité sans surprise, tout en continuant à désirer l'aventure... Dans son cas généralement l'aventure commence le vendredi soir et se termine le lundi matin. Cendrillon va toujours au bal, mais elle n'oublie jamais de rentrer avant minuit; un carrosse est toujours plus souhaitable qu'une citrouille.

L'impératif économique semble dicter à cette catégorie de femmes une ligne de conduite calquée directement sur le discours officiel actuel. On peut facilement accepter que les femmes à l'instar des hommes subissent et intègrent les valeurs à la mode qui sont les conséquences de la prépondérance idéologique du libéralisme économique, tel qu'actuellement véhiculé, et qui cherche à niveler la valeur des citoyens au niveau du simple consommateur. À la limite, et au nom des principes de liberté de conscience et d'action, on ne devrait même pas y trouver à redire.

La liberté des citoyens porte, en extension, sur le droit d'adhérer à un modèle économique et/ou social et sur le droit même d'en faire la promotion, ou à tout le moins d'exprimer son adhésion.

Sauf que, lorsque l'expression des idées personnelles est supportée par la machine médiatique étatique et qu'elle prend une dimension de propagande, la liberté d'expression doit aussi s'étendre au droit de répliquer, de contester et éventuellement de dénoncer de tout citoyen. Quand le discours officiel, cherchant à pénétrer les milieux où une certaine idéologie différente pourrait éventuellement faire son nid et prospérer, dispose des ressources d'un État, il devient relativement aisé de recruter les porte-parole les mieux appropriés pour véhiculer les messages. Quoi de mieux que des femmes, qui plus est des femmes qui se targuent de parler au nom du mouvement féministe, pour organiser la contre-révolution face à une idéologie qui vient changer les règles du jeu traditionnelles, en proposant des modèles nouveaux impliquant l'établissement nécessaire d'une nouvelle assise socio-économique?

Dans le cas précis de Radio-Canada, où l'employeur est en même temps l'État, les impératifs politiques et idéologiques sont nécessairement des critères d'embauche. La valeur du message à émettre est également nécessairement conditionnée par la valeur et la crédibilité de l'émetteur. On cherche ainsi à permettre l'expression d'une certaine pensée, en apparence concordante avec l'idée révolutionnaire, puisque prétendant y adhérer et parler en son nom, dans des limites, pas toujours évidentes, fixées d'entrée de jeu par les technocrates du pouvoir. On verrait ainsi le système traditionnel, d'ailleurs dénoncé à grands cris, sélectionner lui-même ses détracteurs. Il serait pour le moins 
paradoxal qu'il se fasse qu'un système travaille lui-même à sa destruction, fournissant à ses adversaires ses propres armes. On peut donc déjà, ne seraitce qu'à titre de vérification d'ordre logique, douter de la crédibilité idéologique d'une personne à qui l'État traditionnel fournit un large auditoire afin de propager des idées qui mèneraient, si elles étaient suffisamment bien implantées dans les esprits, à son remplacement pur et simple. L'État patriarcal fournirait ainsi ses ressources au mouvement féministe, au nom de la liberté d'action et d'expression, motivé par la grandeur d'âme et l'ouverture d'esprit, afin d'accélérer sa propre mise au rancart. On peut se permettre de demeurer sceptique face à cette prétendue ouverture d'esprit. En effet, si elle était réelle, elle se traduirait par des réalisations autrement plus concrètes qu'une simple tribune médiatique, qui prend ainsi plutôt l'allure d'une tentative d'apaiser des esprits qui pourraient avoir certaines velléités contestataires.

Sous le prétexte de laisser une place à l'expression de la pensée féministe, on sélectionne ainsi des personnes que l'État juge, selon ses propres critères, aptes à la transmettre. Sous l'apparence de la liberté d'expression se cache ainsi une intention de contrer les effets éventuels de la propagation d'une idéologie dérangeante. On peut facilement répliquer que de telles affirmations peuvent être le simple fruit d'une imagination particulièrement délirante et que l'État dans lequel nous vivons n'oserait jamais même penser à commettre une telle exaction. Il n'est pas dans notre intention de faire le procès de toute une société, ou d'entretenir sans fondement l'idée que l'État puisse délibérément s'acharner dans une tentative, savamment orchestrée, d'aliénation des classes laborieuses. Il n'est pas non plus dans notre intention de faire une analyse exhaustive du contenu de chacune des émissions « dites » à caractère féministe de la radio d'État. À titre indicatif, et afin d'éclairer ponctuellement notre discussion, nous ne citerons que certains extraits de l'émission « Et quoi encore » animée par Mme Bazzo et diffusée le jeudi 26 mars 1992, extraits qui reflètent bien l'esprit général véhiculé par le genre de programmes que nous voulons dénoncer.

Ce jour-là, nous retrouvons Marie-France Bazzo et Geneviève SaintGermain, dans le cadre de la « Chronique néo-poupoune » habituelle, discutant des revues ou magazines destinés aux motocyclistes (Moto-Journal, Easy Rider, Biker Magazine, etc.). Parlant de certaines photos que l'on retrouve souvent dans ce genre de publications et montrant généralement des femmes dans des situations ou des poses pour le moins «spéciales », on nous sert les jugements suivants : « Nous autres, on est peut-être néo-poupounes mais, elles, elles sont carrément poupounes ». Doit-on entendre ici que le fait d'être néo-poupounes marque une élévation notable du statut de la femme ou de son rôle dans la société?

Or : en voyant cela, on se rend compte que le féminisme a encore du travail à faire, on est mieux avec «Elle Québec ». Tout en convenant qu'il reste une immense tâche à accomplir pour l'établissement d'une véritable société égalitaire, peut-on réellement considérer que la revue Elle Québec est un 
véhicule approprié pour l'expression d'un féminisme vraiment révolutionnaire?

Parlant des prix des accessoires et des pièces de motos, et plus particulièrement des amortisseurs, on entendit : « Nos petits pots sont moins chers que ça ». Existe-t-il une relation fondant un discours d'émancipation dans le fait que des amortisseurs pour motos soient plus dispendieux que des produits de beauté?

Pour conclure, on nous annonce de façon guillerette que « demain nous parlerons de beauté et de poupounerie comme à l'habitude, à bientôt ». Peuton vraiment entendre ici la promesse de lendemains meilleurs dans un discours aussi édifiant?

D'un point de vue théorique, nous pouvons apprécier qu'il s'agit ici d'une résurgence, sinon d'une réitération, d'une certaine vision des préoccupations fondamentales du monde féminin qui n'est pas sans rappeler celle des années 1950-ı66. On semble opérer une forme de retour aux valeurs dites féminines de façon traditionnelle. La petite bourgeoise n'est plus confinée, de façon absolue, au cadre de la maison familiale. Ses horizons se sont élargis, mais les stéréotypes demeurent. Le confort matériel et l'aisance intellectuelle y gagnent mais nous constatons que l'aliénation est toujours la même. Le conservatisme change de visage, mais même le meilleur lifting ne changera jamais le contenu fondamental. Nous retrouvons toujours une certaine idéalisation du pouvoir de consommer, réservé à une certaine classe relativement aisée, et présentée comme un accomplissement.

La néo-poupoune représente la nouvelle version de la bourgeoise au foyer qui tient à son confort, qu'elle ressent d'ailleurs comme une libération. En entretenant le mythe que la consommation systématique permet l'épanouissement de la personnalité, la néo-poupoune sert adéquatement le nouveau Dieu-dollar. Assoyant son confort personnel sur la quête continuelle de la majorité de la population, elle entretient le clivage social sur lequel repose le nouveau libéralisme économique qui est le credo actuel du pouvoir. En présentant un modèle artificiel, et de toute façon impossible à atteindre, elle prend les commandes d'un courant qui sert à détourner toute une génération des enjeux réels et fondamentaux. La société n'est ainsi plus à refaire, elle devient une sorte d'arène où tout peut se jouer et où l'avenir est ouvert aux seuls gagnants.

Une certaine catégorie de femmes, en l'occurrence les néo-poupounes, se rend ainsi complice du système traditionnel qui cherche, par tous les moyens mis à sa disposition, à se maintenir. Un certain nombre de changements ont été accomplis. Les valeurs tendent à vouloir transiter vers de nouveaux lieux où l'égalité, entre autres, aura vraiment droit de cité. Un certain mouvement a été amorcé, la roue tourne déjà. Mais des gens, des femmes notamment, refusent le changement. En privé, c'est leur droit, mais en public et de surcroît sur une tribune médiatique, cela devient d'une certaine façon indécent. En tant qu'homme (mâle) qui prend lentement conscience de la valeur et des 
incidences des changements en cours, je dis que cette situation est inacceptable. En tant qu'homme, je refuse ce retour en arrière qu'on voudrait nous voir opérer. Et surtout, en tant qu'homme, je refuse par mon silence de me rendre complice de cet enlisement dans des valeurs d'un autre temps. 